\title{
AN IMAGE DEBLURRING METHOD USING DIFFRACTION GRATINGS
}

\author{
G.G. YANG and E. LEITH \\ Department of Electrical and Computer Engineering, The University of Michigan, Ann Arbor, MI 48109, USA
}

Received 16 September 1980

\begin{abstract}
A method of image deblurring is described, in which the restoration filter is derived from Ronchi rulings. Although two-dimensional spatial filtering is used, the method employs a line source, resulting in significant noise reduction. Experimental results are given.
\end{abstract}

\section{Introduction}

This paper has two major aims; to show a method for making image deblurring masks from simple Ronchi ruling diffraction gratings, and second, to show a simple means for introducing noise-reducing redundancy into an optical processing system when the processing is a one-dimensional operation, as in the case of deconvolution of a one-dimensionally blurred image.

Yu has described a method for introducing such redundancy by the use of point source white light [1]. He used diffraction gratings to disperse a white light source into spectral components that travel different paths in the optical system. The signal $s(x)$ is processed and the additional dimension, $y$, is used for wavelength dispersion. He showed that a spatial filter of the form $H\left(f_{x}, y\right)$ can be constructed for one dimensional processing, where, as a function of $f_{x}$, the filter is just the required structure, and as a function of $y$, the filter retains the same form but changes in scale to accommodate the wavelength variation, since each $y=$ constant section of the filter receives a different wavelength.

A similar system was described by Goedgebuer and Gazeu [2] in which prisms are used for dispersion and subsequent undispersion of the light. In either case, the filter must vary by a scaling factor along the $y$ direction.

The method we describe for introduction of redundancy employs an old procedure, the use of an incoherent line source, along with some refinements.

Holographic complex spatial filters are typically made by recording, with a coherent beam, the Fourier transform of an object distribution. The object is of course then the impulse response of the spatial filter. This procedure assumes that one has available at the beginning, as a transparency, the impulse response. This is normally the case with matched filters, but is generally not the case otherwise. Alternatively, one may use computer methods to produce the filter, but the method is more cumbersome. For image deblurring, the impulse response is typically not available as a photographic transparency; thus, one must resort to other means to produce a deblurring filter.

It has been previously shown that a deblurring function can be generated from simple Ronchi rulings by means of spatial filtering [3]. We show here that appropriate deblurring functions (either impulse response or transfer functions) can be generated in other ways from Ronchi rulings.

\section{Theory}

The deblurring of a one-dimensionally blurred image can be formulated as the convolution $u=s * h$

where $s$ is the blurred image and $h$ is the restoring function. Alternatively, eq. (1) can be written 
$u=\mathcal{F}-1\{S H\}$,

where $S$ and $H$ are, respectively, the Fourier transforms of $s$ and $h$, and $\mathcal{F}^{-1}$ means inverse Fourier transformation. Thus, we can restore the image by optically convolving the blurred image with a mask $h$, or by spatial filtering it with a mask $H$. The end result is the same, al though the implementations are different, the former being known as a spatial domain synthesis, and the latter as a frequency domain synthesis.

Various deblurring functions have been described. A simple one, very well suited to our requirements, given by Slepian [4] and developed further by Cutrona et al. [5], is

$h=\sum_{n=1}^{N}[\delta(x-n l)-\delta(x-n l-\Delta)]$.

Such a mask is shown in fig. 1 , where the delta functions have been approximated by rect functions. Also shown in fig. 1 is a heuristic explanation (after Swindell) [6] of the deblurring process when implemented as a convolution operation, $h * s$. Here, $s$ is a rect function of width $l$, which represents an original point of line object, smeared over a width $l$ by the blurring process. If one mentally convolves $s$ and $h$, which can be readily done by inspection, the result is that shown at the bottom of fig. 1. Evidently, the process gives rise to two deblurred images, and in order that the two not overlap, the total length $N l$ of the blurring mask should be as great as the width of the image to be deblurred. Also, unless the mask $h$ is

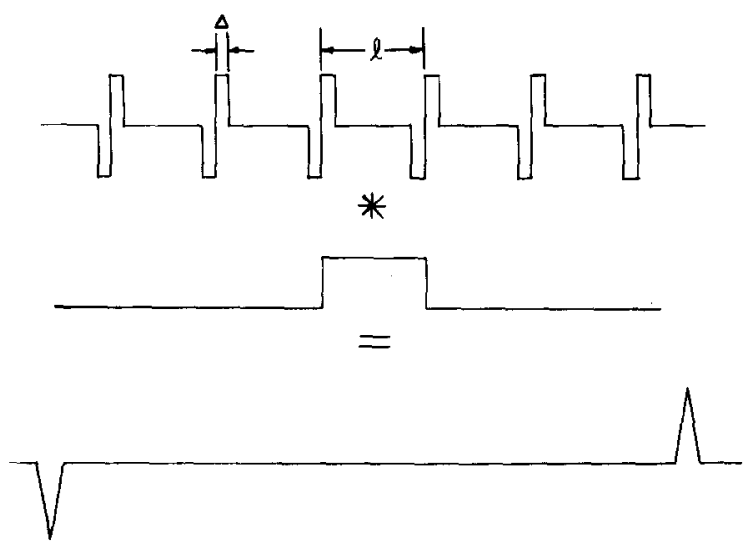

Fig. 1. The deblurring process. quite well made, there will be weaker secondary images, resulting from incomplete cancellation of the contributions of adjacent pulse components of $h$ to the convolution integral.

\section{Implementation}

Our goal is to obtain a deblurring spatial filter $H$, so as to implement the process described heuristically in fig. 1, but as a spatial filter operation rather than as a convolution. By describing the deblurring process in the manner of fig. 1, a simple filter construction method is immediately suggested. A Ronchi ruling of spatial frequency $f_{0}$,

$\frac{1}{2}+\frac{1}{2} \operatorname{sgn}\left(\cos 2 \pi f_{0} y\right)$,

where

$$
\begin{aligned}
\operatorname{sgn} x & =1 \quad x>0, \\
& =-1 \quad x<0,
\end{aligned}
$$

is overlain on a mask

$$
\sum_{n=1}^{N} \operatorname{rect}\left(\frac{x-n l}{\Delta}\right)
$$

consisting of $N$ slits of width $l$. This overlay is photographed. Next, the Ronchi ruling is transported $1 / 2$ of a period in the $y$ direction and the slit mask is transported by $\Delta$ in the $x$ direction, and a second exposure is made, resulting in the mask shown in fig. 2. Such a mask can be regarded as a hologram of the function $h$ given by eq. (3), with the delta function

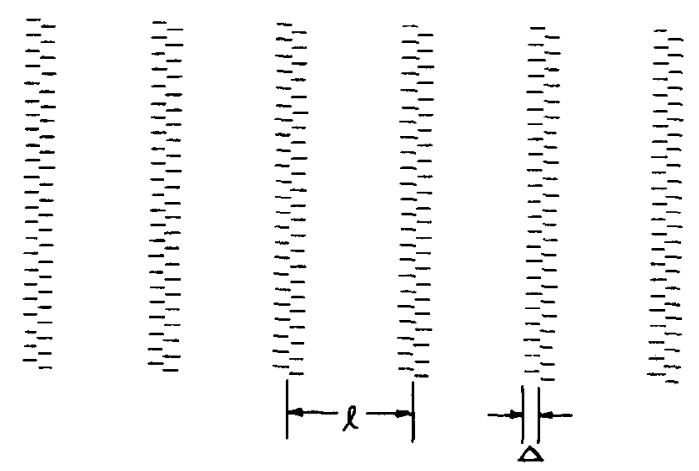

Fig. 2. Mask bearing deblurring impulse function $h$. 


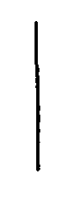

LS
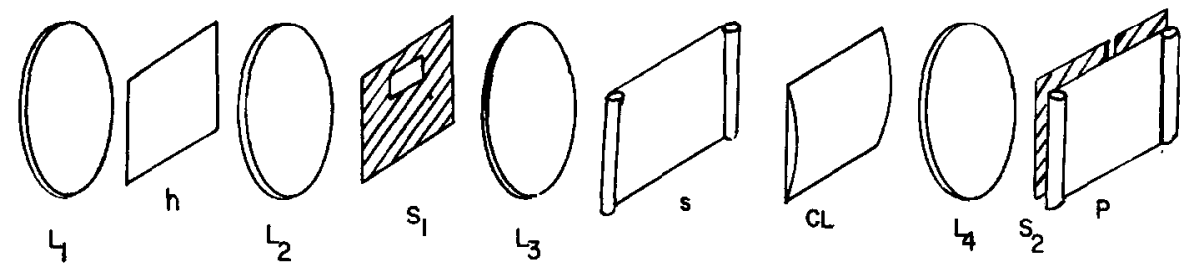

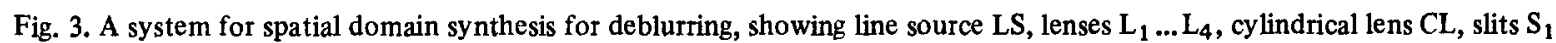
and $S_{2}$, blurred images and recording plane $P$.

being approximated by a rect function of width $\Delta$. Either of the two first diffracted orders, when used to reimage the grating mask, is just the restoration function $h$ we require. The two first diffracted orders are the same and either can be used. Similarly, the third or any other odd order would in theory also serve. Such a mask, if imaged after spatial filtering onto the blurred image, can be used to implement the deblurring convolution $s * h$.

In a manner similar to the method described in ref. [3], the resulting optical processor, in fig. 3 , could be used with white light, since the only spatial filtering required is to separate the first diffracted order from the others, and this can always be done in white light, since over the full visible spectrum, the first order will be completely separated from all other orders. Similarly, a line source of monochromatic or polychromatic light can be used, provided the source is not so long that the diffracted orders overlap. Evidently, the higher the spatial frequency $f_{0}$ the more spatially incoherent can be the source. Coherence is required only for the $x$ direction, which is the direction of the blur. It is technically much easier to put the spatial carrier in the $y$ direction, as we have done, for various reasons, and it is significant that placing the carrier in the $y$ direction makes very little demand on either temporal coherence or $y$-directed spatial coherence. For the $x$-dimension, no temporal coherence at all is required, for reasons explained in ref. [3]. Of course, spatial coherence in $x$ is necessary.

However, from our viewpoint here the mask $h$ is only an intermediate step in our goal, which is to generate the spatial filter $H$. One has only to record as a hologram the lens-produced Fourier transform of $h$ (using one 1st diffracted order of $h$ ). The Fourier transform thus generated consists of a sequence of points at spatial frequency coordinates $f_{x}=1 / l, 2 / l, \ldots$, and $f_{y}=0$, with each point having a width $\Delta f_{x}=1 / N l$.

We require the hologram to be of extended length in the $f_{y}$ direction; thus we introduce a cylindrical lens in the spatial filter construction system, and the points become lines extended in $f_{y}$ (fig. 4). In recording the hologram, it is desirable to introduce the spatial carrier in the $f_{y}$ direction since the line width may, depending on $H$, be rather fine, requiring a very

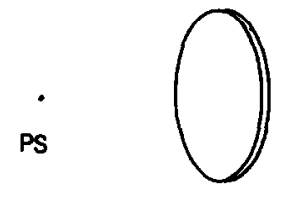

LI

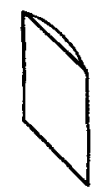

CL
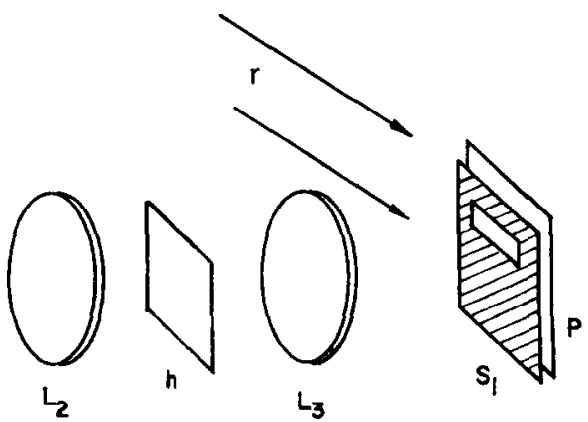

Fig. 4. Filter-making system, showing lenses $L_{1}, L_{2}$, cylindrical lens $C L$, mask $h$, with diffracted orders $0,+1,-1$, with 0 and -1 being blocked out by mask $P$. Reference beam $r$, incident from top, records holographically the first diffracted order from $h$. 
fine fringe pattern in $f_{x}$ to give adequate sampling of the lines.

A more challenging method of filter construction is to make the source incoherent in the $f_{y}$ direction since then the filter-producing process will have noisereducing properties. We do not describe here in detail how to do this; we note, however, that the theory given in ref. [7] can be adapted for this purpose. Especially noteworthy is that the reference beam can be introduced so as to produce a spatial carrier along $f_{y}$ just as before, even though the light is spatially incoherent in the $y$ dimension. This possibility arises because the grating interferometer described in ref. [7] produces high contrast, high spatial frequency fringes even with spatially incoherent illumination.

\section{Use of the filter}

The spatial filter may be used in the standard way, shown in fig. 5 . The source is a line in which all points are incoherent. This line is then imaged through $s$ onto the spatial filter $H$. The spatial frequency spectrum is smeared in $f_{y}$, but preserved in $f_{x}$. Each element of the source projects the data through the optical system in a different direction, and forms the Fourier transform $S$ centered at a different vertical position on the filter $H$. The filter thus diffracts some of the light into a pair of first orders, while some remains undiffracted. At the output three images are formed, with the top and bottom ones being the convolution $s * h$ and the cross correlation $s * h$, respectively; since the function $h$ is real and symmetrical, $s * h$ and $s * h$ are not distinguishable and either can serve as the output. The undiffracted order leads essentially to a reimaging of $s$. The spatial carrier on the filter must be sufficiently large as to separate the three output images and is not dependent on the length of the source. Thus, the source can be of a length limited only by the field of the lens. In this way, a redundancy is achieved comparable to that obtained previously $[1,2]$, when a point source of white light was used in combination with gratings or prisms to achieve a similar redundancy effect; the different wavelengths are then transmitted through the system in different directions and form $S$ at different vertical positions at the filter $H$. The present method has two advantages relative to the white light method:

1) The various channels developed in this alternative way are separated from the source onward instead of only from the dispersing grating onward, resulting in better noise suppression.

2) The spatial filter need be of one dimensional variation only, whereas in the white light case the filter must be scaled as a function of $f_{y}$.

An alternative way to use the filter is described with reference to fig. $3 ; h$ is removed and replaced with the line source LS. The filter $H$ is Fourier transformed to yield the impulse response $h$. Again, one of the two diffracted orders is used, and the signal $s$ is overlain on the image $h$ and moved across the aperture along the $x$ direction. A combination cylindrical and spherical lens forms the Fourier transform of the product $h(x) s\left(x^{\prime}-x, y\right)$ (where $x^{\prime}$ is the displacement of $s)$ :

output $=u_{0}=\int h(x) s\left(x^{\prime}-x, y\right) \exp \left(\mathrm{j} 2 \pi x x_{\mathrm{f}} / \lambda F\right) \mathrm{d} x$,

where $F$ is the focal length of the final spherical lens, and $x_{\mathrm{f}}$ is the coordinate of the output plane. We place a slit at $x_{\mathrm{f}}=0$, recording then the light distribution

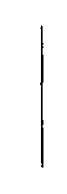

LS

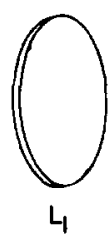

4
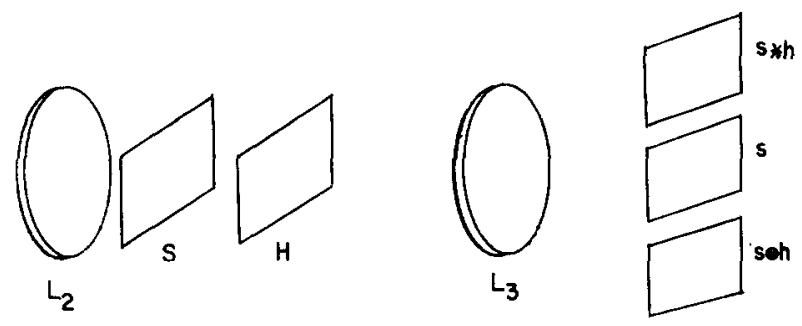

Fig. 5. Spatial filtering system for deblurring, showing line source $L S$, lenses $L_{1}, L_{2}$, and $L_{3}$, blurred image s and filter $H$. Output consists of three terms. 
in the slit as $s$ is moved through the aperture, producing

$u_{0}=\int h(x) s\left(x^{\prime}-x, y\right) \mathrm{d} x=h * s$,

which is the same result as previously given, except now the convolution is given as a function of time along a line, instead of all at one time over a twodimensional spatial distribution. Since we again use a line source, we again achieve the redundancy of the previous method. Additional redundancy is available if the light source is polychromatic.

\section{Experimental results}

The optical system for the experiment is essentially that shown in fig. 5 . The line source must have the various points along its length incoherent with each other. To generate the light source, a cylindrical lens was placed as shown in fig. 6 , causing a line of light to form. This line is the source for the processing system. To make the elements of the line source mutually incoherent, a rotating ground glass was placed along the line image. It was found that some of the light fell outside the line image. Therefore, a slit was placed just after the rotating ground glass.

Experimental results are shown in fig. 7. Fig. 7(a) shows a blurred image. The blur which was chosen to be 10 line widths, a line being the width of the lines that constitute the letters in the unblurred object. The deblurred image is shown in fig. 7(b), when a line source was used, and again in fig. 7(c) when a point source is used. The noise reduction resulting from the line source is considerable.

In theory, two images should be observed, whereas in the experiment, three major images are observed, with the top one being imperfectly deblurred. The
PS

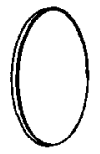

L

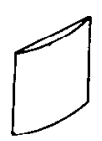

$\mathrm{CL}$

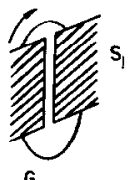

Fig. 6. Creation of an incoherent line source from a laser beam, using point source $P S$, lens $L_{1}$, cylindrical lens $C L$, rotating ground glass $G$ and slit $S_{1}$. Line image, $G$, and $S$ are ideally all in the same plane.
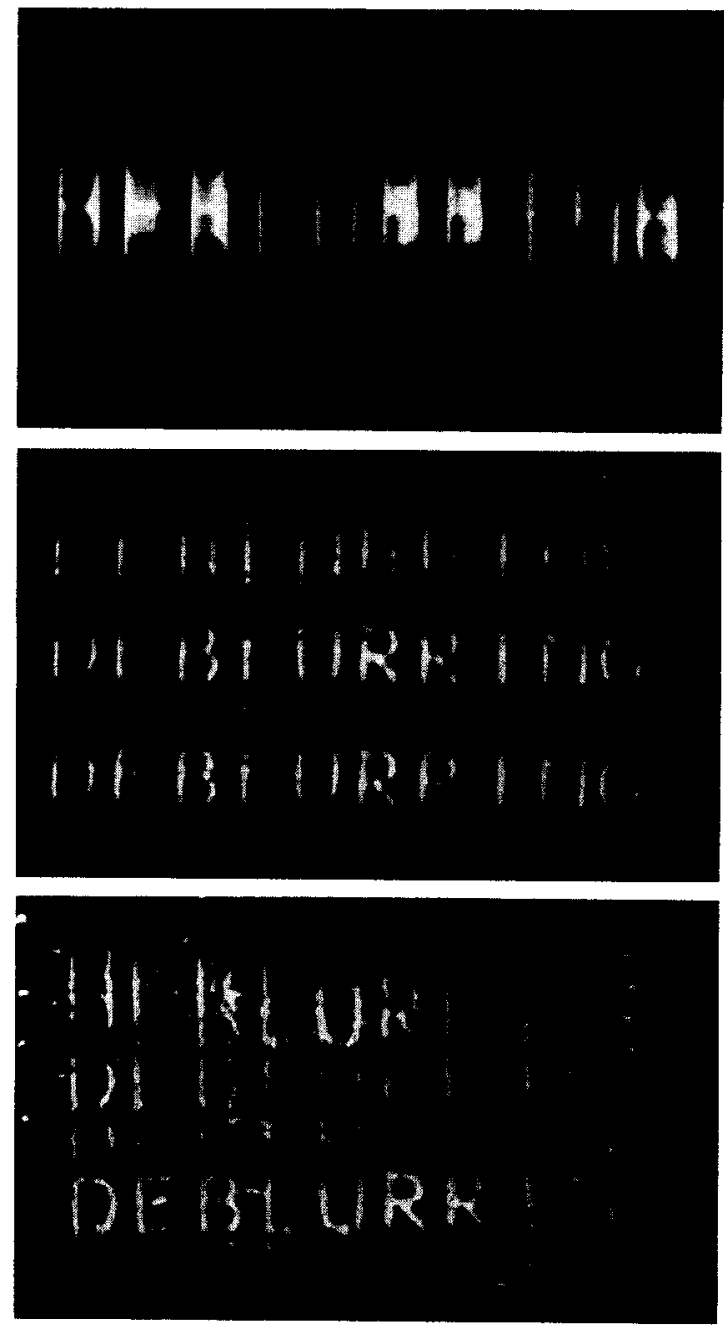

Fig. 7. Experimental results. Top, blurred image; center, deblurred with incoherent line source; bottom, deblurred with point source.

reason for this additional image is presently not known. Also seen are some weaker residual images, resulting from imperfect filter balance, the possibility of which we have previously noted. Careful matching of the filter and spectrum can minimize these extraneous responses, as indeed we have done. Their complete elimination is difficult. We note that the optical arrangement shown in fig. 5 enables the scale of the Fourier transform $S$ to be altered, through axial movement of the blurred image. 
This work was supported in part by the Air Force Office of Scientific Research (grant AFOSR 77-3356).

\section{References}

[1] F.T.S. Yu, Appl, Optics 17 (1978) 3571.

[2] J.P. Goedgebuer and R. Gazeu, Optics Comm. 27 (1978) 53.
[3] E. Leith, Optics Lett. 5 (1980) 70.

[4] D. Slepian, On the restoration of photographs blurred by image motion, Woods Hole Summer Study Report on Restoration of Atmospherically Degraded Images, July 1966.

[5] L.J. Cutrona and W.D. Hall, Some considerations in post-facto blur removal, Proceedings of seminar, Evalua tion of motion-degraded images, Dec. 1968.

[6] W. Swindell, App1. Optics 9 (1979) 2459.

[7] E.N. Leith and G.J. Swanson, Appl. Optics 19 (1980) 638. 